\title{
El proceso de secado por atomización: formación de gránulos y cinética de secado de gotas
}

\author{
ROSA MONDRAGÓN ${ }^{*}$, J. ENRIQUE JULIA', ANTONIO BARBA², JUAN CARLOS JARQUE² \\ 'Departamento de Ingeniería Mecánica y Construcción. \\ ${ }^{2}$ Departamento de Ingeniería Química e Instituto Universitario de Tecnología Cerámica. \\ Universitat Jaume I. Campus de Riu Sec. 12071 Castellón de la Plana. España \\ *correspondencia/corresponding author: e-mail: rosa.mondragon@uji.es
}

\begin{abstract}
El proceso de secado por atomización es una operación unitaria que interviene en numerosas aplicaciones industriales. Para cada aplicación en concreto, el material granulado resultante debe poseer unas propiedades determinadas que dependen de las condiciones en las que se lleve a cabo el secado por atomización, y cuya dependencia debe conocerse para así optimizar la calidad del material obtenido. El gran número de variables que influyen en los procesos de transferencia de materia y energía que tienen lugar, y en la formación del material granulado, han hecho necesario el análisis en detalle del proceso de secado. A lo largo de los años han sido muchos los estudios realizados sobre el secado por atomización y la influencia de las variables del proceso en la cinética de secado y las propiedades del material granulado obtenido, para todo tipo de materiales. En este artículo se recogen los trabajos más importantes publicados correspondientes tanto al secado por atomización como al secado de gotas individuales, así como los estudios cuyo objetivo es el modelado de la cinética de secado de gotas.
\end{abstract}

Palabras clave: Secado, Suspensiones, Microestructura, Modelización.

Spray Drying Processing: granules production and drying kinetics of droplets

Spray drying is a unit operation very common in many industrial processes. For each particular application, the resulting granulated material must possess determined properties that depend on the conditions in which the spray drying processing has been carried out, and whose dependence must be known in order to optimize the quality of the material obtained. The large number of variables that influence on the processes of matter and energy transfer and on the formation of granular material has required a detailed analysis of the drying process. Over the years there have been many studies on the spray drying processing of all kind of materials and the influence of process variables on the drying kinetics of the granulated material properties obtained. This article lists the most important works published for both the spray drying processing and the drying of individual droplets, as well as studies aimed at modeling the drying kinetics of drops.

Keywords: Drying, Suspensions, Microstructure, Modeling.

\section{INTRODUCCIÓN}

El proceso de secado por atomización es una operación básica que consiste en la transformación de una suspensión o disolución en un material seco particulado, mediante la atomización del primero en un medio caliente y seco (1). El secado por atomización de gotas es utilizado en muchas aplicaciones industriales de los sectores cerámico, químico, alimentario, farmacéutico.

Cada proceso industrial y uso posterior del polvo atomizado obtenido requiere de unas propiedades determinadas, que sean óptimas para la aplicación en cuestión. Por ejemplo, en industria farmacéutica se desea producir gránulos huecos y porosos que actúen como portadores de fármacos (2). Por el contrario, para optimizar el proceso de proyección por plasma de materiales, es necesario hacer uso de gránulos de elevada densidad y compacidad (3). En el caso de la industria de detergentes, la microestructura interna y la porosidad de los gránulos también debe ser la adecuada para permitir la retención de agentes activos en su interior (4).
Las características del polvo atomizado resultante del proceso de secado por atomización vienen determinadas, para cada instalación industrial, por varios factores. Dentro de las variables que influyen en el proceso se puede distinguir entre aquellas propias de la suspensión: densidad de la suspensión, contenido en sólidos, viscosidad, tensión superficial, temperatura de la suspensión, estado de desfloculación y distribución de tamaños de partícula de las materias primas; y las del equipo de secado: temperatura, caudal y humedad relativa del aire de secado, presión de inyección y el diámetro de salida del inyector.

Debido al elevado número de variables que influyen en la cinética de secado y en las propiedades del producto final se pueden obtener materiales granulados de características muy diferentes en función de las condiciones experimentales bajo las cuales se lleve a cabo el proceso de secado. Es por esto que resulta de gran interés el hecho de poder controlar, 
cuantificar y modelar la influencia que ejerce cada variable en la cinética de secado y en las propiedades finales del gránulo, y así producir materiales de características óptimas para cada aplicación en cuestión. De las etapas presentes en el proceso de secado por atomización, es durante la evaporación cuando tienen lugar todas las transformaciones físico-estructurales que confieren al gránulo la microestructura y geometría final y por lo tanto determina sus propiedades físicas. Es por esto que el estudio de dicha etapa resulta de gran interés con el fin de poder controlar y predecir las características del material resultante.

\section{PROCESO DE SECADO DE GOTAS}

\subsection{Variación de la humedad de la gota}

Cuando una gota con gran cantidad de líquido entra en contacto con el aire caliente, ésta experimenta un proceso de secado que pude dividirse en varias etapas tal y como se muestra en la Figura $1(1,5-8)$. Inicialmente, la gota experimenta un aumento de calor sensible hasta la temperatura húmeda correspondiente a la temperatura del aire de secado (tramo $0-1)$. En ese instante se inicial el primer periodo de secado o periodo de velocidad de secado constante (tramo 1-2). En esta etapa, el líquido se desplaza desde el interior de la gota lo suficientemente rápido como para mantener la superficie saturada de humedad. Como consecuencia, la evaporación de líquido se produce en la superficie y la tasa de evaporación iguala a la de una gota formada por líquido puro. Es en esta fase en la que se produce la contracción de la gota, cuyo diámetro al cuadrado varía de forma lineal con el tiempo (ley del $d^{2}$ ). Durante este periodo, la velocidad de secado permanece constante y su valor es el máximo alcanzado en todo el proceso. Si las condiciones ambientales de secado no varían, la temperatura de la gota permanece igual a la temperatura húmeda del aire en todo el intervalo de tiempo.

En un determinado momento, se alcanza la humedad crítica (punto 3) en la que la migración de líquido ya no es capaz de mantener la superficie saturada. La concentración de líquido en la superficie de la gota se reduce a un valor mínimo y se inicia el segundo periodo de secado o periodo de velocidad de secado decreciente (tramo 3-5). En él, las partículas primarias forman una costra de aglomerados sumergida en el líquido. En el secado posterior, la costra aflora a la superficie de la gota y se transforma en un costra seca, determinando el diámetro final del gránulo que deja de contraer. La gota puede considerarse como un gránulo húmedo de diámetro constante formado por dos zonas: la costra sólida de estructura porosa, y el núcleo húmedo formado por líquido y sólido. La costra formada genera una resistencia adicional a la evaporación del líquido, que depende de la capacidad de difusión de los fluidos a su través. A medida que transcurre el proceso y el espesor de la costra aumenta, disminuye la velocidad de secado. Al formarse la costra y dejar la superficie de estar saturada de humedad, la temperatura del gránulo dejará de ser igual a la temperatura húmeda del aire y aumentará progresivamente hasta alcanzar la temperatura de secado. Dependiendo del material a secar, este periodo puede dividirse a su vez en subperiodos (tramos 2-3 y 3-4).

El proceso de secado continua hasta que el gránulo tiene el contenido en humedad final deseado. Si el tiempo de secado es suficientemente largo, esta humedad final será igual a la mínima humedad posible, que es igual al contenido de humedad de equilibrio con el aire de secado.

\subsection{Cambios microestructurales}

A medida que transcurre el proceso de secado y se modifica la humedad de la gota, tienen lugar una serie de transformaciones que confieren al gránulo unas determinadas características morfológicas y microestructurales. Durante el primer periodo de secado, debido a que la energía superficial de la interfase sólido-vapor es mayor que la correspondiente a la interfase líquido-vapor, las partículas se desplazan hacia el interior de la gota para minimizar la energía superficial (2, 9). Esta migración de sólido favorece que la superficie de la gota se encuentre saturada de humedad prolongándose el primer periodo de secado. Si la velocidad de reordenación de partículas hacia el centro de la gota es mucho más lenta que el secado, se alcanzará una situación en la que la superficie no puede conservar la condición de saturación y las partículas

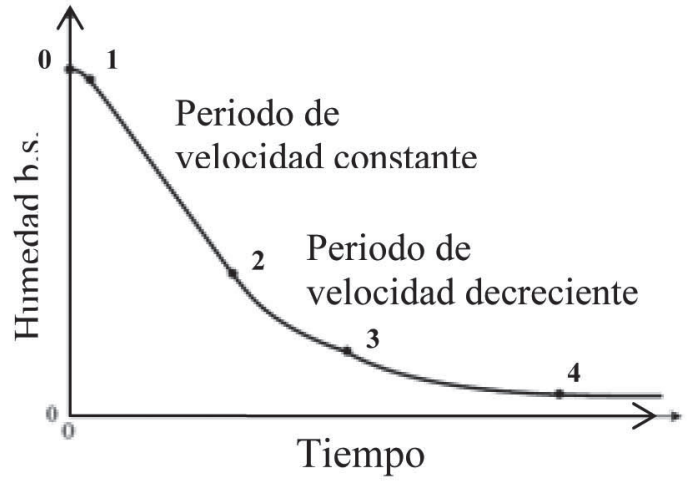

(a)

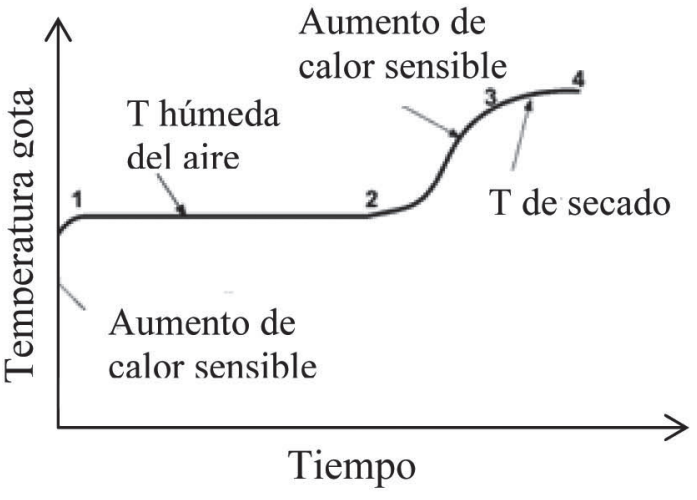

(b)

Figura 1. (a) Variación de la humedad con el tiempo. (b) Evolución de la temperatura de la gota durante el secado. 


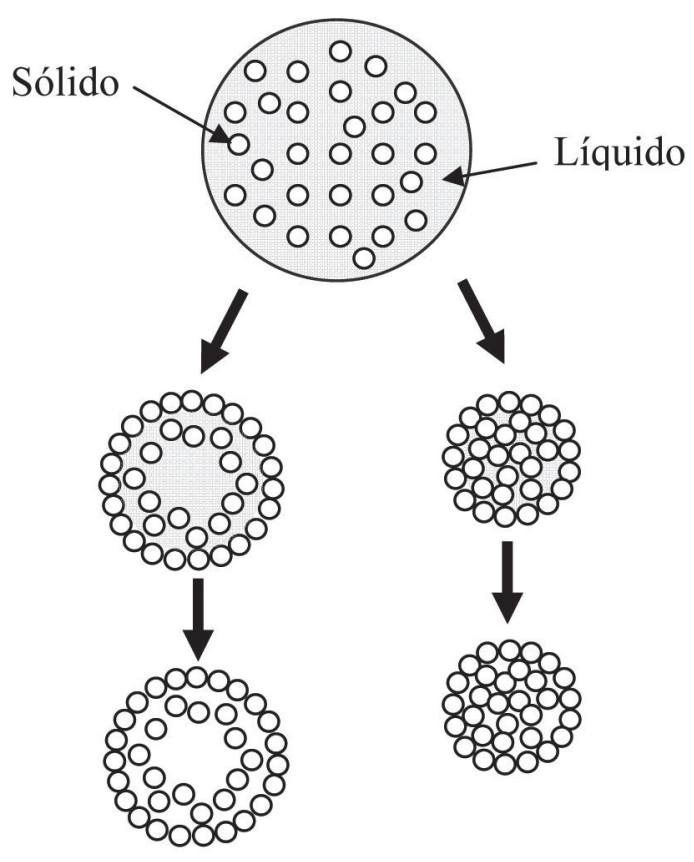
(a) $P e>>1$
(b) $P e<<1$

Figura 2. Formación de gránulos (a) huecos y (b) compactos durante el proceso de secado.

colapsan formando una costra. A partir de ese instante el gránulo deja de contraer, se inicia el segundo periodo de secado y la humedad presente en el gránulo corresponde a la humedad crítica.

A medida que la costra empieza a formarse se genera una fuerza capilar debido a los meniscos de líquido entre partículas que hacen que éstas se aproximen y se mantengan unidas en la superficie (10). Por otra parte, cuando una cantidad pequeña de agua se evapora se transmite un flujo de calor desde la superficie de la gota hasta el aire. En la superficie se crean gradientes locales de temperatura que causan el desplazamiento termoforético de las partículas hacia la interfase líquido-vapor debido al enfriamiento por evaporación, provocando la acumulación de partículas en la interfase $(11,12)$. De esta forma, durante el segundo periodo de secado el retroceso del plano de evaporación provoca un aumento de la densidad de partículas en la superficie, y la costra crece progresivamente desde la superficie del gránulo hacia el interior como resultado de la incorporación progresiva de partículas a la costra.

La formación de gránulos huecos con poro central o gránulos compactos depende de que las partículas tengan la capacidad y el tiempo necesario para reordenarse $(2,9,13)$. La medida cuantitativa de la capacidad de secado viene dada por el número de Peclet, $P e$, el cual representa la importancia relativa del tiempo necesario para que las partículas se reordenen $\left(r_{g}^{2} / D_{s}\right)$ con respecto del tiempo de secado $\left(t_{f}\right)$ :

$$
P e=\frac{r_{g}^{2}}{t_{f} \cdot D_{S}}
$$

donde $r$ es el radio de la gota, $t$ es el tiempo de secado de la gota y $D_{S}$ es la difusividad del sólido en el interior de la gota que puede calcularse a partir de la ecuación de StokesEinstein:

$$
D_{S}=\frac{k_{B} \cdot T_{g}}{6 \cdot \pi \cdot \eta \cdot r_{p}}
$$

donde $k_{B}$ es la constante de Boltzmann, $T_{8}$ es la temperatura de la gota, $\eta$ es la viscosidad de la suspensión y $r_{p}$ es el radio de las partículas.

Si el número de Peclet es muy superior a $1, P e>>1$, el secado es mucho más rápido que la velocidad de difusión de las partículas hacia el centro de la gota y por lo tanto, el tiempo empleado en evaporar el líquido es mucho menor que el necesario para que la gota se homogeneíce. Como consecuencia, el colapso de las partículas en la superficie se producirá antes, a una humedad crítica superior, dando lugar a la formación de gránulos huecos con costra (Figura 2a). Por el contrario, si el número de Peclet es muy inferior a 1, $P e<<1$, el proceso de secado se considera lento. En este caso, las partículas tienen tiempo suficiente para difundirse hacia el interior de la gota, de forma que cuando el material sólido colapsa y se inicia el segundo periodo, las partículas forman una estructura sólida compacta sin presencia de poro central (Figura 2b).

\section{INVESTIGACIÓN REALIZADA EN EL SECADO POR ATOMIZACIÓN}

A lo largo de los años han sido varios los estudios realizados sobre la influencia de variables en el proceso de secado por atomización, llevados a cabo todos ellos con suspensiones de distinta naturaleza, y bajo diversas condiciones experimentales (Tabla I). En todos los casos se utilizaron atomizadores o nebulizadores a escala reducida que permitían controlar algunas de las variables relacionadas con el proceso de secado y obtener, en algunos casos, correlaciones generales entre las variables del proceso y las propiedades finales de los gránulos.

El secado por atomización es un proceso industrial de alta complejidad en el que se producen fenómenos de atomización de chorros, fenómenos de transporte de materia y energía entre las gotas y el aire, interacciones entre las gotas y la pared del atomizador, etc. Las primeras investigaciones sobre el proceso de secado por atomización se remontan a principios de los años 50 (14-19). La relación entre las condiciones de operación y las características del polvo atomizado se estudiaron para líquidos puros, soluciones acuosas (nitrato amónico, sulfato sódico, silicato sódico, cloruro sódico) y suspensiones de tipo alimentario (leche en polvo, café). A partir de los años 80 estos estudios se extendieron al uso de materiales utilizados en la industria cerámica como son $\mathrm{SiO}_{2^{\prime}} \mathrm{Al}_{2} \mathrm{O}_{3^{\prime}}, \mathrm{ZrO}_{2}$ y $\mathrm{Si}_{3} \mathrm{~N}_{4}$ (20-25). En estos estudios se obtuvieron correlaciones que relacionaban las propiedades de las suspensiones (contenido en sólidos, temperatura y presencia de aditivos) con las propiedades finales del polvo atomizado obtenido (morfología, microestructura, densidad, resistencia mecánica y fluidez).

Recientemente, los materiales nanoparticulados han recibido mucha atención debido a sus propiedades eléctricas, ópticas, magnéticas y mecánicas que los diferencian del resto. El proceso de secado por atomización permite obtener gránulos nanoestructurados de mayor tamaño de gran interés 
TABLA I. ESTUDIOS DE SECADO POR ATOMIZACIÓN DE DISOLUCIONES Y SUSPENSIONES DE MICROPARTÍCULAS.

\begin{tabular}{|c|c|c|c|c|}
\hline Ref. & Materiales & $\begin{array}{c}\text { Temperatura, } \\
T\left({ }^{\circ} \mathrm{C}\right)\end{array}$ & $\begin{array}{c}\text { Contenido en solidos } \\
\text { másico, } \\
Y_{S}(\%)\end{array}$ & Propiedades analizadas \\
\hline $\begin{array}{c}\text { Ranz and Marshall, } 1952 \\
(14,15)\end{array}$ & $\begin{array}{l}\text { Agua, benceno, aniline, } \\
\text { sales solubles, leche }\end{array}$ & 220 & - & $\begin{array}{l}\text { Tasas de evaporación, variación } \\
\text { de diámetro con el tiempo } \mathrm{d}=\mathrm{f}(\mathrm{t})\end{array}$ \\
\hline $\begin{array}{l}\text { Duffie and Marshall, } 1953 \\
(16,17)\end{array}$ & $\begin{array}{c}\text { Colorantes, silicato sódico, } \\
\text { cloruro sódico }\end{array}$ & $300-540$ & $12,5-19,5$ & Densidad del polvo atomizado \\
\hline $\begin{array}{l}\text { Crosby and Marshall, } \\
1958(18)\end{array}$ & Sulfato sódico, café, arcilla & $300-540$ & $7-22$ & $\begin{array}{l}\text { Distribución de tamaños de } \\
\text { gránulo y densidad del polvo } \\
\text { atomizado }\end{array}$ \\
\hline $\begin{array}{l}\text { Charlesworth and } \\
\text { Marshall, } 1960 \text { (19) }\end{array}$ & $\begin{array}{l}\text { Sulfato sódico, nitrato } \\
\text { amónico, cloruro, café }\end{array}$ & 160 & $5-50$ & Tasas de evaporación \\
\hline $\begin{array}{c}\text { Lukasiewicz et al., } \\
1989(20)\end{array}$ & Polvo cerámico & - & $50-75$ & $\begin{array}{l}\text { Morfología, microestructura y } \\
\text { densidad del polvo atomizado }\end{array}$ \\
\hline Takahashi et al., 1995 (21) & Nitruro de silicio & - & 23 & $\begin{array}{c}\text { Densidad del polvo atomizado y } \\
\text { resistencia mecánica }\end{array}$ \\
\hline Walker et al., 1999 (22) & Alúmina & 200 & $60-70$ & $\begin{array}{l}\text { Influencia de aditivos en la } \\
\text { morfología y densidad del polvo } \\
\text { atomizado }\end{array}$ \\
\hline Bertrand et al., 2003 (23) & Alúmina & 180 & 60 & $\begin{array}{c}\text { Distribución de tamaños de } \\
\text { gránulo, densidad, morfología, } \\
\text { microestructura y fluidez del } \\
\text { polvo atomizado }\end{array}$ \\
\hline Bertrand et al., 2005 (24) & Alúmina, Zircona & $100-150$ & $30-60$ & $\begin{array}{l}\text { Influencia de } \mathrm{pH} \text { en morfología y } \\
\text { densidad del polvo atomizado }\end{array}$ \\
\hline Zainuddin et al., 2010 (25) & Alúmina & 200 & 60 & $\begin{array}{l}\text { Porosidad } \\
\text { Microestructura } \\
\text { resistencia }\end{array}$ \\
\hline
\end{tabular}

TABLA II. ESTUDIOS DE SECADO POR ATOMIZACIÓN DE SUSPENSIONES DE NANOPARTíCULAS.

\begin{tabular}{|c|c|c|c|c|}
\hline Ref. & Materiales & $\begin{array}{c}\text { Temperatura, } \\
T\left({ }^{\circ} \mathrm{C}\right)\end{array}$ & $\begin{array}{c}\text { Contenido en } \\
\text { solidos másico, } \\
Y_{S}(\%)\end{array}$ & Propiedades analizadas \\
\hline Hadinoto et al., 2006 (2) & Sílice & $100-150$ & $0,18-1,76$ & $\begin{array}{l}\text { Compacidad y tamaño de los } \\
\text { gránulos, formación de costra }\end{array}$ \\
\hline $\begin{array}{l}\text { Hadinoto and Cheow, } 2009 \\
\text { (9) }\end{array}$ & Sílice & $110-130$ & $0,2-1,2$ & $\begin{array}{l}\text { Influencia de } \mathrm{pH} \text {, contenido } \\
\text { en sólidos y temperatura en la } \\
\text { formación de costra }\end{array}$ \\
\hline Sen et al., 2010 (10) & Sílice & 160 & 5 & $\begin{array}{l}\text { Influencia de la concentración } \\
\text { de electrolito en la formación de } \\
\text { gránulos }\end{array}$ \\
\hline Iskandar et al., 2003 (11) & Sílice & $200-1000$ & 1 & $\begin{array}{c}\text { Control de la morfología de los } \\
\text { gránulos }\end{array}$ \\
\hline Sen et al., 2009 (12) & Alúmina & 160 & $1-5$ & $\begin{array}{l}\text { Influencia de la velocidad } \\
\text { de secado en la morfología y } \\
\text { microestructura de los gránulos }\end{array}$ \\
\hline Bahadur et al., 2010 (13) & Sílice & 160 & $2,5-20$ & $\begin{array}{l}\text { Distribución de tamaños y } \\
\text { morfología de los gránulos }\end{array}$ \\
\hline Fair and Lange, 2004 (27) & Alúmina & - & 60 & $\begin{array}{l}\text { Distribución de tamaños de } \\
\text { gránulo }\end{array}$ \\
\hline Tsapis et al., 2005 (28) & Poliestireno & - & 0,4 & $\begin{array}{l}\text { Elasticidad de la costra y } \\
\text { deformación de los gránulos }\end{array}$ \\
\hline Wang et al., 2005 (29) & Sílice & $200-1000$ & $0,04-4$ & $\begin{array}{c}\text { Morfología y estado } \\
\text { de aglomeración de las } \\
\text { nanopartículas }\end{array}$ \\
\hline Sen et al., 2007 (30) & Sílice & 160 & 5 & $\begin{array}{l}\text { Influencia de la velocidad } \\
\text { de secado en la morfología y } \\
\text { microestructura de los gránulos }\end{array}$ \\
\hline Kim et al., 2008 (32) & Sílice & 150 & $0,05-10$ & $\begin{array}{l}\text { Morfología y microestructura de } \\
\text { los gránulos }\end{array}$ \\
\hline
\end{tabular}

que permiten su uso en diversos campos como catálisis, cromatografía, pigmentos, cerámicas avanzadas, farmacología, etc (26). En el campo de los materiales nanoestructurados se han realizado estudios de secado por atomización que han permitido relacionar las propiedades del gránulo con las de la suspensión (2, 9-12, 13, 27-31) (Tabla II). En este caso, se incorporan como variables de la suspensión aquellas propias de la ciencia coloidal como son el $\mathrm{pH}$ del medio, la presencia 
de electrolitos y las fuerzas de atracción y repulsión presentes entre partículas.

En todos los casos se utilizaron atomizadores o nebulizadores a escala reducida que permitían controlar algunas de las variables relacionadas con el proceso de secado por atomización permitiendo obtener, en algunos casos, correlaciones generales entre las variables del proceso y las propiedades finales de los gránulos. Sin embargo, en los equipos empleados no es posible controlar totalmente los parámetros del proceso de secado. Los fenómenos de transferencia de materia y energía se complican debido a variaciones en el sistema global de secado. Por una parte, la transferencia de calor depende de las diferencias de temperaturas cuya distribución en los gases de secado es difícil de predecir. Además, si la distribución de tamaños de gotas es amplia la velocidad de secado varía de unas gotas a otras de forma que las más pequeñas se secan más rápidamente que el resto. Estas variaciones de los valores locales de temperatura, diámetro de gota, etc., dentro del chorro, dificultan el análisis de los resultados. Finalmente, los fenómenos físicos involucrados en estos procesos son de una gran complejidad y, por lo tanto, es necesario realizar un análisis más detallado. Es por todo esto que resulta de mayor interés centrar el estudio en el secado de gotas individuales que pueden ser aisladas, de forma que puede analizarse de forma más precisa el efecto que una determinada variable ejerce en el proceso de secado sin verse afectado por posibles interacciones.

\section{INVESTIGACÓN REALIZADA EN EL SECADO DE GOTAS INDIVIDUALES}

\subsection{Equipos y técnicas de secado de gotas individuales}

Para estudiar el proceso de secado de gotas individuales y su cinética es necesario disponer de un sistema que permita suspender una única gota sometida a una corriente de aire caliente, y poder registrar la evolución de su forma, tamaño y masa.

Los primeros estudios realizados con gotas individuales se llevaron a cabo utilizando la técnica del filamento (Figura 3). En esta técnica, la gota a secar es suspendida en el extremo de un filamento de vidrio. La evolución de la morfología de la gota se registra mediante técnicas de visualización, mientras que la evolución de la masa de la gota se registra a partir de la desviación que sufre el filamento. A medida de la gota se seca

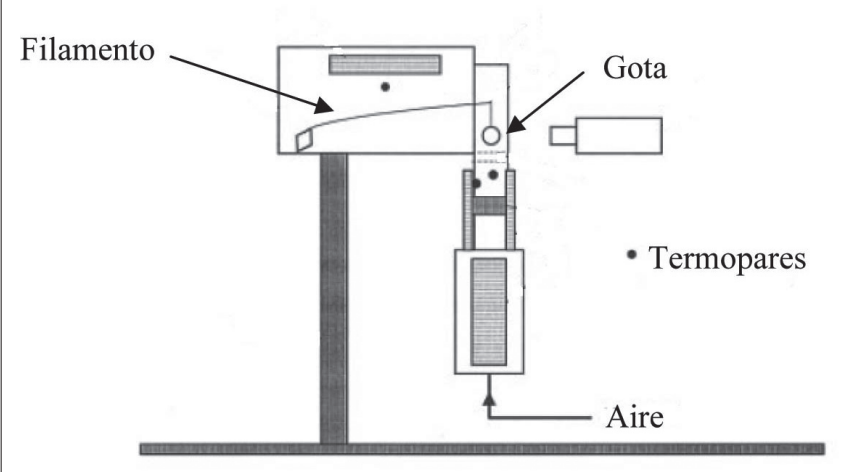

Figura 3. Técnica del filamento (65). y su masa disminuye, la posición del filamento que la sostiene varía con respecto de su posición inicial. Esta desviación es directamente proporcional a la masa de la gota y su relación se obtiene mediante una calibración previa. La principal desventaja que presenta esta técnica es que la gota no se encuentra totalmente aislada, sino que está en contacto directo con el filamento. En el punto de unión de la gota con el filamento, la evaporación se ve afectada por la presencia de éste.

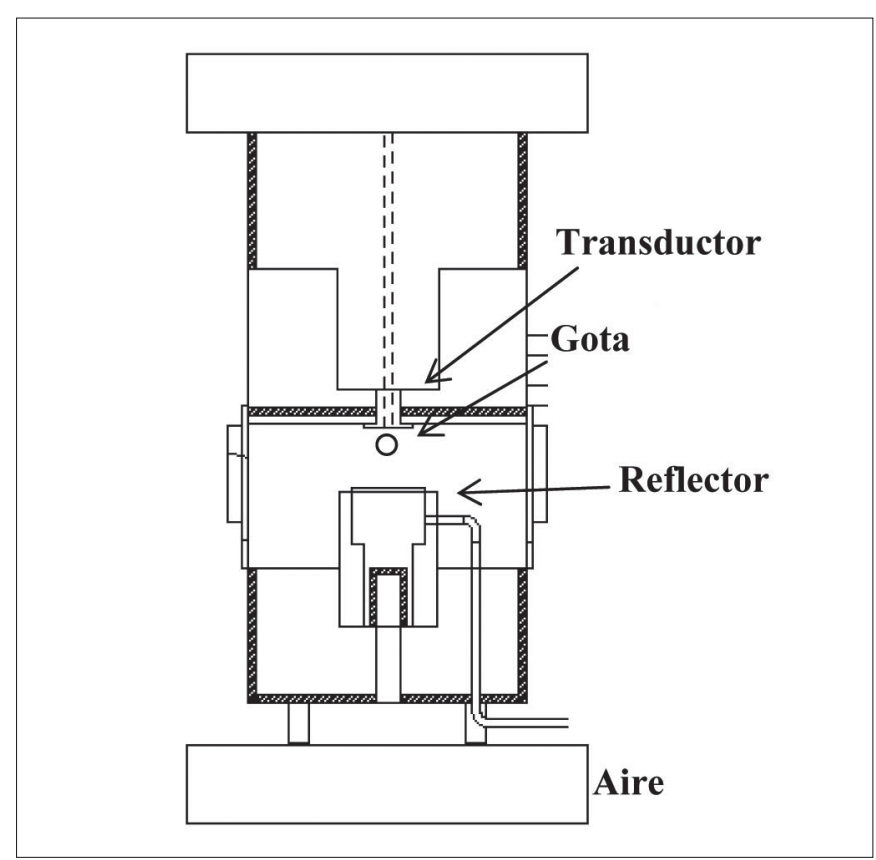

Figura 4. Levitador acústico (45).

En las últimas décadas, el uso de levitadores (acústicos, ópticos, electrodinámicos y aerodinámicos) ha mostrado algunas ventajas con respecto de los métodos convencionales de suspensión de gotas debido a la ausencia de contacto mecánico con la gota (32). De todos ellos, los levitadores acústicos (o de ultrasonidos) presentan una complejidad experimental baja y permiten trabajar con prácticamente cualquier tipo de líquido en un rango amplio de diámetros de gotas (desde $0,1 \mathrm{~mm}$ hasta varios milímetros). Tiene la ventaja de que la muestra no requiere de ninguna propiedad física especial (carga eléctrica o índice de refracción determinados) y además, proporciona una posición estable de la gota. Como se puede observar en la Figura 4, el levitador acústico consta de un transductor piezoeléctrico y un resonador cóncavo situados de forma que entre ellos se genera una campo acústico. Este campo da lugar a una serie de nodos de alta y baja presión de forma que cuando una gota es introducida dentro del campo acústico, ésta permanece estable en una posición correspondiente a un nodo de baja presión, debido al balance entre la fuerza gravitatoria y la fuerza de presión del nodo. Esta técnica permite registrar, mediante técnicas de visualización, la evolución de la morfología de la gota, su volumen y su posición. Estos datos permiten obtener la evolución de la humedad de la gota con el tiempo.

\subsection{Investigación realizada en el secado de gotas individuales en levitadores acústicos}

En los últimos años los levitadores acústicos han sido utilizados ampliamente para estudiar el secado de gotas de 
TABLA III. EstUdIOS DE SECADO DE GOTAS INDIVIDUALES EN LEVITADOR ACÚSTICO.

\begin{tabular}{|c|c|c|c|c|c|}
\hline Ref. & Materiales & $\begin{array}{c}\text { Temperatura, } \\
T\left({ }^{\circ} \mathrm{C}\right)\end{array}$ & $\begin{array}{l}\text { Contenido en } \\
\text { solidos másico, } \\
\qquad Y_{S}(\%)\end{array}$ & $\begin{array}{c}\text { Volumen } \\
\text { inicial de la } \\
\text { gota, } \\
V_{0}(1)\end{array}$ & Propiedades analizadas \\
\hline Kastner et al., 2001 (5) & $\begin{array}{c}\text { Suspensión acuosa de } \\
\text { vidrio }\end{array}$ & 22 & $5-20$ & 0,5 & $\begin{array}{c}\text { Periodos de secado y tasas } \\
\text { de evaporación }\end{array}$ \\
\hline $\begin{array}{l}\text { Kastner et al., } 2000(34, \\
\text { 35) }\end{array}$ & $\begin{array}{c}\text { Suspensión acuosa de } \\
\text { vidrio }\end{array}$ & 23 & $5-30$ & $0,5-1,5$ & $\begin{array}{c}\text { Tasas de evaporación y porosidad } \\
\text { media del gránulo }\end{array}$ \\
\hline Kastner et al., 2001 (36) & $\begin{array}{c}\text { Suspensión acuosa de } \\
\text { vidrio }\end{array}$ & $22-50$ & $5-20$ & $0,5-3$ & Tiempos de secado \\
\hline Yarin et al., 2002 (37) & n-hexadecano & 25 & - & - & Deformación de la gota. \\
\hline Yarin et al., 2002 (38) & $\begin{array}{c}\text { Suspensión acuosa de } \\
\text { vidrio }\end{array}$ & $23-84$ & $5-30$ & $0.26-1.02$ & $\begin{array}{c}\text { Variación de diámetro y posición } \\
\text { de la gota. Tiempo de secado }\end{array}$ \\
\hline $\begin{array}{l}\text { Schiffter and Lee, } 2007 \\
\qquad(39,40)\end{array}$ & $\begin{array}{l}\text { Agua. Disoluciones } \\
\text { acuosas de manitol, } \\
\text { trehalosa y catalasa }\end{array}$ & $25-60$ & 10 & $1,5-2,5$ & $\begin{array}{c}\text { Tamaño de la gota. Coeficientes } \\
\text { de transferencia }\end{array}$ \\
\hline $\begin{array}{l}\text { Wulsten and Lee, } 2008 \\
\text { (41) }\end{array}$ & Agua & $25-60$ & - & - & $\begin{array}{c}\text { Tamaño y temperatura de la gota. } \\
\text { Coeficientes de transferencia }\end{array}$ \\
\hline Kumar et al., 2010 (42) & Nanofluidos de sílice & $20-80$ & $0,2-10$ & 0,1 & $\begin{array}{l}\text { Morfología y temperatura } \\
\text { de la gota }\end{array}$ \\
\hline Saha et al., 2010 (43) & Nitrato de cerio & $20-80$ & - & 0,1 & $\begin{array}{l}\text { Morfología y temperatura } \\
\text { de la gota }\end{array}$ \\
\hline Saha et al., 2011 (44) & Nanofluidos de sílice & $20-80$ & 30 & 0,1 & Particle Image Velocimetry (PIV) \\
\hline $\begin{array}{c}\text { Mondragón et al., } 2011 \\
(45)\end{array}$ & $\begin{array}{c}\text { Suspensión acuosa de } \\
\text { vidrio }\end{array}$ & $60-120$ & $1-50$ & $0,1-0,7$ & $\begin{array}{c}\text { Tasas de evaporación, tamaño } \\
\text { y porosidad del gránulo }\end{array}$ \\
\hline $\begin{array}{c}\text { Mondragón et al., } 2012 \\
(46)\end{array}$ & $\begin{array}{l}\text { Suspensión de gres } \\
\text { porcelánico }\end{array}$ & $70-100$ & $65-70$ & $0,4-0,7$ & $\begin{array}{l}\text { Tasas de evaporación, tamaño, } \\
\text { porosidad y resistencia mecánica }\end{array}$ \\
\hline $\begin{array}{l}\text { Mondragón et al., 2012, } \\
2013(47,48)\end{array}$ & Nanofluido de sílice & $80-120$ & $2-20$ & $0,3-0,8$ & $\begin{array}{c}\text { Cinética de secado, propiedades } \\
\text { mecánicas, empaquetamiento } \\
\text { de partículas }\end{array}$ \\
\hline
\end{tabular}

líquidos puros, líquidos multicomponentes, y suspensiones sólido-líquido (Tabla III).

A finales de los años 90, Yarin et al. (33) estudió en profundidad las características del campo acústico formado en el interior del levitador y el efecto que ejerce sobre la gota y su secado. A partir de entonces, empezaron a surgir trabajos de secado de gotas formadas por mezclas binarias de líquidos (agua-metanol y agua-etanol) y suspensiones de micropartículas de vidrio en agua, en los que se establecen las técnicas para la determinación de la porosidad de los gránulos, los periodos de secado existentes y su duración y las tasas de evaporación correspondientes a cada uno de los periodos (5, 34-38). Todos los estudios mencionados fueron llevados a cabo a temperatura ambiente en su mayoría, debido a la limitación de temperatura que presenta el transductor piezoeléctrico, el cual no soporta más de $60{ }^{\circ} \mathrm{C}$ de temperatura.

Posteriormente, se realizaron estudios de secado de gotas de agua pura y suspensiones de compuestos orgánicos (manitol, trehalosa...) a $60{ }^{\circ} \mathrm{C}$, máxima temperatura permitida por la instalación experimental (39-41). En ellos, además de obtener la variación del diámetro de la gota con el tiempo y las tasas de evaporación, se obtuvo la evolución de la temperatura de la gota mediante termografía de infrarrojos.

En los últimos años, se ha desarrollado una nueva técnica que permite el secado de gotas en el levitador a temperaturas elevadas, mediante el calentamiento local de la gota con un láser de $\mathrm{CO}_{2}$ (42-44). Hasta el momento se han realizado estudio de secado de gotas de nitrato de cerio y nanopartículas de sílice, a $80^{\circ} \mathrm{C}$ de temperatura. En estos estudios se ha analizado la evolución de la morfología de la gota, su diámetro, y se ha aplicado termografía de infrarrojos para seguir la evolución de la temperatura de la gota. Además se ha aplicado la técnica de PIV (Particle Image Velocimetry) para obtener la distribución de velocidades del sólido dentro de la gota. Sin embargo, el uso de radiación láser requiere de un control preciso de la velocidad de calentamiento, ya que un calentamiento rápido da lugar a una evaporación explosiva de la gota como resultado de la formación de puntos calientes.

Para evitar el uso de una fuente de calor externa, y que el único aporte de calor a la gota sea el del aire de secado (condiciones más próximas a las que se desarrolla el secado por atomización) Mondragón et al., (45) realizaron una serie de modificaciones en un levitador comercial de forma que, con la nueva configuración pudieron realizar ensayos a temperaturas elevadas de hasta $120^{\circ} \mathrm{C}$, más próximas a las encontradas industrialmente. La puesta a punto de la instalación se realizó con suspensiones de vidrio en agua, analizándose la evolución del tamaño de gota, las tasas de evaporación y la porosidad media final del gránulo. Los mismos autores, haciendo uso del levitador modificado, llevaron a cabo estudios de secado de gotas de suspensiones utilizadas en la industria cerámica conteniendo sólidos de tamaño micrométrico (46), estudios de secado de gotas de nanofluidos $(47,48)$ y secado de gotas de suspensiones conteniendo mezclas de sólidos de tamaño nanométrico y micrométrico $(49,50)$. En ellos se analizó la influencia de las condiciones de secado (contenido en 
sólidos, pH y concentración de electrolitos de la suspensión, y temperatura de secado y volumen inicial de la gota) en la cinética de secado, la resistencia mecánica de los gránulos y la fracción de empaquetamiento de las partículas en el interior de la costra. Se estableció un procedimiento que permite evaluar el grado de compacidad de los gránulos a partir de las curvas de secado y la fracción de empaquetamiento de los sólidos, obtenida mediante el modelado de la variación de la viscosidad de la suspensión con el contenido en sólidos.

\section{MODELADO DE CURVAS DE SECADO}

Los modelos de cinética de secado de gotas existentes pueden clasificarse en diferentes categorías. Generalmente existen tres tipos de aproximaciones para la formulación de los modelos:

1. Modelos que emplean simultáneamente las ecuaciones diferenciales de conservación de materia y energía haciendo uso de las ecuaciones y coeficientes de difusión.

2. Modelos basados en una aproximación semiempírica que utiliza el concepto de curva de secado característica (CDC).
Esta curva característica tiene en cuenta los diferentes periodos de secado (periodo de velocidad de secado constante y decreciente).

3. Modelos basados en una aproximación a la ingeniería de la reacción, REA, (Reaction Engineering Approach).

Hasta la fecha han sido muchos los materiales estudiados. Los modelos de difusión, más tradicionales, se han aplicado a todo tipo de materiales mientras que los modelos CDC y REA, más recientes, se han empleado mayoritariamente para el modelado del secado de productos de la industria alimentaria, y más recientemente para secado de gotas de nanofluidos. En la Tabla IV se resumen los principales trabajos de distintos autores. Los resultados teóricos han sido comparados con resultados experimentales obtenidos del secado de gotas individuales, haciendo uso de la técnica de suspensión del filamento de vidrio o el levitador acústico.

\subsection{Modelos de difusión}

El modelo de difusión ha sido ampliamente utilizado para predecir la velocidad de secado. El primer trabajo en que se utilizó el modelo para secado de gotas fue publicado en los

TABLA IV. APLICACIÓN DE LOS MODELOS DE SECADO A DISTINTOS MATERIALES.

\begin{tabular}{|c|c|c|c|}
\hline Ref. & Material & Modelo de secado & $\begin{array}{l}\text { Técnicas de obtención de datos } \\
\text { experimentales }\end{array}$ \\
\hline Ranz and Marshall, $1952(14,15)$ & $\begin{array}{l}\text { Agua, Benceno } \\
\text { Nitrato amónico } \\
\text { Cloruro sódico } \\
\text { Leche desnatada }\end{array}$ & Difusión & Filamento \\
\hline $\begin{array}{l}\text { Charlesworth and Marshall, } 1960 \\
\text { (19) }\end{array}$ & $\begin{array}{l}\text { Sulfato sódico } \\
\text { Nitrato amónico } \\
\text { Café }\end{array}$ & Difusión & Filamento \\
\hline Sano and Keey, 1982 (51) & Leche desnatada & Difusión & Filamento \\
\hline Fyhr and Kemp, 1998 (52) & $\begin{array}{l}\text { Madera, Arcilla } \\
\text { Gel de sílice } \\
\text { Resinas }\end{array}$ & Difusión, CDC & Estudio teórico \\
\hline Langrish and Kockel, 2001 (60) & Leche & $\mathrm{CDC}$ & Filamento \\
\hline Chen and Lin, 2005 (65) & $\begin{array}{l}\text { Leche desnatada } \\
\text { Leche entera }\end{array}$ & CDC, REA & Filamento \\
\hline Patel and Chen, 2005 (58) & $\begin{array}{l}\text { Leche desnatada } \\
\text { Leche entera }\end{array}$ & CDC, REA & Filamento \\
\hline Lin and Chen, 2006 (66) & Lactosa & REA & Filamento \\
\hline Lin and Chen, 2007 (67) & $\begin{array}{c}\text { Crema en polvo } \\
\text { Concentrado de proteínas }\end{array}$ & REA & Filamento \\
\hline Mezhericher et al., 2007 (68) & $\begin{array}{l}\text { Leche desnatada } \\
\text { Sílice coloidal }\end{array}$ & Difusión, REA & Filamento \\
\hline Mezhericher et al., 2008 (69) & Leche entera & Difusión, REA & Filamento \\
\hline Woo et al., 2008 (61) & $\begin{array}{c}\text { Sacarosa } \\
\text { Maltodextrina }\end{array}$ & CDC, REA & Filamento \\
\hline Chen, 2008 (64) & Leche desnatada & REA & Filamento \\
\hline Handscomb et al., 2009 (54) & $\begin{array}{l}\text { Detergente } \\
\text { Sílice coloidal }\end{array}$ & Difusión & Filamento \\
\hline Handscomb et al., 2009 (55) & $\begin{array}{l}\text { Sílice coloidal } \\
\text { Sulfato sódico }\end{array}$ & Difusión & Filamento \\
\hline Patel et al., 2009 (70) & $\begin{array}{c}\text { Sacarosa } \\
\text { Maltodextrina } \\
\end{array}$ & REA & Filamento \\
\hline Handscomb and Kraft, 2010 (56) & Sílice coloidal & Difusión & Filamento \\
\hline Fu et al., $2011(71)$ & Lactosa & REA & Filamento \\
\hline Mezhericher et al., 2011 (57) & Sílice coloidal & Difusión & Filamento \\
\hline Mondragón et al., $2013(48,49)$ & Nanofluidos de sílice & REA & Levitador acústico \\
\hline
\end{tabular}


años 50 y desde entonces se ha empleado para modelar el comportamiento en secado de numerosos materiales $(14,15$, 19, 51-57). La fuerza impulsora para el transporte de agua es el gradiente de humedad entre el aire y la gota/gránulo. Dicho transporte se produce por difusión del agua líquida o vapor de agua. El agua se difunde a través del sólido y se evapora en la superficie. Cuando se alcanza la humedad crítica y se forma la costra porosa, el plano de evaporación se desplaza hacia el interior del gránulo y el vapor de agua ha de difundirse a través de la costra.

La distribución temporal y espacial de los componentes durante el secado se obtiene resolviendo la ecuación de difusión.

$$
\begin{aligned}
\frac{\partial c_{M, i}}{\partial t} & =\nabla \cdot\left(D_{i, j} \nabla c_{M, i}\right) \\
\frac{\partial c_{M, i}}{\partial t} & =\frac{D_{i, j}}{r^{2}} \frac{\partial}{\partial r}\left(r^{2} \frac{\partial c_{M, i}}{\partial r}\right)
\end{aligned}
$$

donde $c_{M, i}$ es la concentración másica del componente $i, D_{i, j}$ es la difusividad del componente $i$ en el componente $j$, y $r$ es el radio de la gota.

En general, el modelo describe el proceso mediante un conjunto de ecuaciones diferenciales ordinarias y en derivadas parciales y sus correspondientes condiciones de contorno.

La resolución es complicada por la existencia de un dominio de contorno que se desplaza como debido a la contracción de la gota en el primer periodo, y al retroceso de la interfase costra-núcleo durante el segundo periodo. Como resultado, la solución numérica de estos modelos requiere de tiempo y recursos de cálculo importantes. Además, requiere el conocimiento de muchos coeficientes de transporte y características del material como coeficientes de difusión de materia y energía, compacidad de las partículas, humedad crítica...bajo distintas condiciones de secado. La difusión de agua puede describirse haciendo uso de una difusividad efectiva. Sin embargo, dicha difusividad tampoco es fácil de determinar con precisión ya que varía con el contenido en agua y requiere conocer el perfil de humedad en el interior del gránulo.

\subsection{Curva de secado característica (CDC)}

El modelo CDC fue formulado asumiendo que la velocidad de secado es una función lineal, de primer orden con respecto al contenido en humedad libre de la gota/ gránulo (58). El modelo se basa en la existencia de dos periodos de secado. El periodo de velocidad de secado constante se asimila a la evaporación de gotas pequeñas de líquido puro, mientras que el periodo de velocidad de secado decreciente se determina multiplicando la velocidad de secado al final del primer periodo por una función que depende de la humedad presente, la humedad de equilibrio y la humedad crítica (59). Esta aproximación supone que, a cada contenido en humedad libre, le corresponde una velocidad de secado específica relativa a la velocidad de secado en el primer periodo, e independiente de las condiciones de secado externas (60). La velocidad de secado puede expresarse como (61):

$$
\frac{d X}{d t}=f \cdot \frac{h_{c} \cdot A}{m_{S} \cdot \Delta H_{v}}\left(T-T_{g}\right)
$$

$$
\begin{array}{ll}
f=\frac{X-X_{e q}}{X_{c r}-X_{e q}} & X \leq X_{c r} \\
f=1 & X>X_{c r}
\end{array}
$$

donde $h_{c}$ es el coeficiente individual de transferencia de energía, $A$ es el área de la gota, $m_{S}$ es la masa de sólido, $H_{v}$ es la entalpía de vaporización del agua y $T$ y $T_{g}$ son las temperaturas del aire y la gota respectivamente.

El modelo es simple y es sencillo matemáticamente. Sin embargo, la aplicación del CDC requiere conocer a priori los valores de la humedad crítica, que han de ser obtenidos experimentalmente y los cuales dependen de las condiciones de secado. En materiales con escaso o nulo periodo de velocidad de secado constante (materiales que no contraen durante el secado), la humedad crítica puede ser sustituida por la humedad inicial, haciendo que este modelo sea más apropiado para este tipo de materiales $(58,62)$.

\subsection{Aproximación a la ingeniería de la reacción (REA)}

El modelo REA considera el proceso de secado como un proceso de activación en el cual tiene que superarse una barrera energética para que tenga lugar la eliminación de humedad (61).

La ecuación matemática que representa el modelo, utiliza el gradiente de concentraciones de vapor como fuerza impulsora para la eliminación de agua de la superficie de la gota. La velocidad de secado puede expresarse de la siguiente manera (63):

$$
\frac{d m_{L}}{d t}=-h_{m} \cdot A \cdot\left[\rho_{v, \text { sup }}-\rho_{v, a}\right]
$$

donde $m_{L}$ es la masa de agua, $\rho_{v, \text { sup }}$ es la concentración de vapor en la superficie de la gota, $\rho_{v, a}$ es la concentración de vapor en el aire de secado, $h_{m}$ es el coeficiente de transferencia de materia y $A$ es el área de la gota.

La concentración de vapor en la superficie, $\rho_{v, s u p^{\prime}}$ es igual a la concentración de vapor saturado, $\rho_{v, s a t^{\prime}}$ cuando la superficie de la gota se encuentra completamente saturada de humedad (primer periodo de secado) y disminuye durante el segundo periodo de secado. Ambas concentraciones se pueden relacionar por medio de la humedad relativa en la superficie de la gota,

$$
\rho_{v, \text { sup }}=\varphi_{\text {sup }} \cdot \rho_{v, \text { sat }}\left(T_{g}\right)
$$

Por otra parte, la humedad relativa de la superficie puede expresarse mediante la siguiente función:

$$
\varphi_{\text {sup }}=\exp \left(-\frac{\Delta E_{V}}{R \cdot T_{g}}\right)
$$

donde $E_{v}$ representa la energía de activación aparente necesaria para el proceso de secado, y refleja el grado de dificultad que supone la eliminación de agua en los diferentes periodos de secado. Cuando el sólido se encuentra completamente cubierto de agua, la energía de activación tiende a valor cero, mientras que aumenta a medida que el contenido en humedad disminuye, debido a la dificultad creciente de eliminar agua para bajos contenidos.

En general, es posible obtener relaciones entre la energía de activación aparente y la humedad, como una propiedad 
característica de cada material bajo distintas condiciones de secado. La energía de activación se obtiene a partir de los resultados experimentales, de la siguiente forma:

$$
\Delta E_{V}=-R \cdot T_{g} \cdot \ln \left(\frac{-\frac{d m_{L}}{d t} \cdot \frac{1}{h_{m} \cdot A}+\rho_{v, a}}{\rho_{v, s a t}}\right)
$$

$\mathrm{Al}$ representar la energía de activación frente al contenido en humedad libre $\left(X-X_{e q}\right)$ se obtiene una función del tipo:

$$
\Delta E_{V}=a \cdot \exp \left[-b \cdot\left(X-X_{e q}\right)^{c}\right]
$$

donde el parámetro $a$ debe ser independiente del contenido en humedad inicial, mientras que $b$ y $c$ están interrelacionados y dependen de la humedad inicial y de la temperatura de secado $(48,64)$.

En los últimos años el modelo ha demostrado una buena concordancia con los resultados experimentales, así como una sencillez y facilidad de cálculo que requiere de escasos recursos matemáticos.

\section{CONCLUSIONES}

Elprocesodesecadoporatomizaciónesutilizadoampliamente en diferentes sectores industriales para obtener materiales granulados con unas propiedades determinadas, a partir del secado de suspensiones. Cada aplicación posterior del polvo atomizado requiere de una morfología y una microestructura adecuadas para cada uso. Todos los estudios llevados a cabo han tenido por objetivo identificar qué condiciones del proceso dan lugar a gránulos esféricos o irregulares, huecos y con elevada porosidad o densos, y con buenas prestaciones mecánicas.

Inicialmente se realizaron estudios en secaderos por atomización a escala, en los que se pudo comprobar la influencia de las condiciones del proceso en las propiedades medias del polvo atomizado. A partir de estos trabajos se pudo inferir que las propiedades más importantes a tener en cuenta eran la temperatura del aire de secado, el tamaño inicial de las gotas, el contenido en sólidos de la suspensión y el tamaño de partícula y estado de aglomeración del material sólido.

Sin embargo, en los secaderos por atomización existen variaciones de temperatura y de tamaños de gota dentro del chorro de material atomizado, lo que dificulta el análisis de los resultados. Por ello, se optó por el estudio de secado de gotas individuales, que permite llevar a cabo el proceso bajo condiciones perfectamente controladas. Mediante el uso de levitadores acústicos, se ha podido cuantificar la influencia ejercida sobre las propiedades del gránulo, de las variables que ya se conocía previamente que influían en el proceso. Además, el secado de gotas individuales ha proporcionado datos experimentales de la cinética de secado que han permitido la validación experimental de los distintos modelos disponibles.

Los resultados más recientes establecen que se puede determinar la compacidad de los gránulos a partir de la cinética de secado, el tamaño final del gránulo y la fracción de empaquetamiento de los sólidos contenidos en la suspensión. Con ello se puede identificar que variables interesa modificar con el fin de obtener el material granulado óptimo para una aplicación concreta.
Estos estudios llevados a cabo de forma genérica con materiales y suspensiones de distinta naturaleza (distinta composición química, tamaño de partícula micrométrico y nanométrico...) pueden utilizarse directamente en situaciones y aplicaciones reales con el fin de aumentar la calidad del producto final y la eficiencia del proceso.

\section{BIBLIOGRAFÍA}

1. K. Masters. Spray Drying Handbook. Longman Scientific and Technical, 1991.

2. K. Hadinoto, P. Phanapavudhikul, Z. Kewu, R.B.H. Tan. Novel formulation of large hollow nanoparticles aggregates as potential carriers in inhaled delivery of nanoparticle drugs. Ind Eng Chem Res 45, 3697-3706, 2006.

3. A. Schrijnemakers, S. Andre, G. Lumay, N. Vandewalle, F. Boschini, R. Cloots, B. Vertruyen. Mullite coatings on ceramic substrates: stabilisation of $\mathrm{Al}_{2} \mathrm{O}_{3}-\mathrm{SiO}_{2}$ suspensions for spray drying of composite granules suitable for reactive plasma spraying. J Eur Ceram Soc 29, 2169-2175, 2009.

4. P. Meenan, K.J. Roberts, U.P.C. Knight, K. Yuregir. The influence of spray drying conditions on the particle properties of recrystallized burkeite $\left(\mathrm{Na}_{2} \mathrm{CO}_{3} \cdot\left(\mathrm{Na}_{2} \mathrm{SO}_{4}\right)_{2}\right)$. Powder Technol 90, 125-130, 1997.

5. O. Kastner, G. Brenn, D. Rensink, C. Tropea. The acoustic tube levitator: a novel device for determining the drying kinetics of single droplets. Chem Eng Technol 24, 335-339, 2001.

6. M. Mezhericher, A. Levy, I. Borde. Heat and mass transfer of single/wet particle drying. Chem Eng Sci 63, 12-23, 2008.

7. M. Mezhericher, A. Levy, I. Borde. Theoretical models of single droplet drying kinetics: a review. Drying Technol 28, 278-293, 2010.

8. M. Mezhericher, A. Levy, I. Borde. Modelling the morphological evolution of nanosuspension droplet in constant-rate drying stage. Chem Eng Sci 66, 884-896, 2011.

9. K. Hadinoto, W.S. Cheow. Hollow spherical nanoparticulate aggregates as potential ultrasound contrast agent: shell thickness characterization. Drug Development and Industrial Pharmacy 35, 1169-1179, 2009.

10. D. Sen, A. Khan, J. Bahadur, S. Mazumder, B.K. Sapra. Use of small-angle neutron scattering to investigate modifications of internal structure in selfassembled grains of nanoparticles synthesized by spray drying. J Colloid Interface Sci 347, 25-30, 2010.

11. F. Iskandar, L. Gradon, K. Okuyama. Control of the morphology of nanostrcutured particles prepared by the spray drying of a nanoparticle sol. J Colloid Interface Sci 265, 296-303, 2003.

12. D. Sen, S. Mazumder, J.S. Melo, A. Khan, S. Bhattyacharya, D.F. D'Souza. Evaporation driven self-assembly of a colloidal dispersion during spray drying: volume fraction dependent morphological transition. Langmuir 25, 6690-6695, 2009.

13. J. Bahadur, D. Sen, S. Mazumder, B. Paul, A. Khan, G. Ghosh. Evaporationinduced self assembly of nanoparticles in non-buckling regime: volume fraction dependent packing. J Colloid Interface Sci 351, 357-364, 2010.

14. W.E. Ranz, J.R. Marshall. Evaporation from drops 1. Chem Eng Progress 48, 141-146. 1952.

15. W.E. Ranz, J.R. Marshall. Evaporation from drops 2. Chem Eng Progress 48, 173-180. 1952

16. J.A. Duffie, W.R. Marshall. Factors influencing the properties of spraydried materials, Part I. Chem Eng Progress 49, 417-423, 1953.

17. J.A. Duffie, W.R. Marshall. Factors influencing the properties of spraydried materials, Part II. Chem Eng Progress 49, 480-486, 1953.

18. E.J. Crosby, W.R. Marshall. Effects of drying conditions on the properties of spray-dried particles. Chem Eng Progress 54, 56-63, 1958.

19. C.H. Charlesworth, W.R. Marshall. Evaporation from drops containing dissolved solids. AIChE J 6, 9-23, 1960.

20. S.J. Lukasiewicz. Spray-drying ceramic powders. J Am Ceram Soc 72, 617$624,1989$.

21. H. Takahashi, N. Shinohara, M. Okumiya. Influence of slurry flocculation on the character and compaction of spray-dried silicon nitride granules. J Am Ceram Soc 78, 903-908, 1995.

22. W.J. Walker, J.S. Reed, S.K. Verma. Influence of slurry parameters on the characteristics of spray-dried granules. J Am Ceram Soc 82, 1711-1719, 1999.

23. G. Bertrand, C. Filiatare, H. Mahdjoub, A. Foissy, C. Coddet. Influence of slurry characteristics on the morphology of spray-dried alumina powders. J Eur Cer Soc 23, 263-271, 2003.

24. G. Bertrand, P. Roy, C. Filiatare, C. Coddet. Spray-dried ceramic powders: a quantitative correlation between slurry characteristics and shapes of the granules. Chem Eng Sci 60, 95-102, 2005.

25. M.I. Zainuddin, S. Tanaka, R. Furushima, K. Uematsu. Correlation between slurry properties and structures and properties of granules. J Eur Cer Soc 30, 3291-3296, 2010.

26. J. Kim, O. Wilhelm, S.E Pratsinis. Nanoparticle packaging. Adv Eng Mater 4, 494-496, 2002. 
27. G.E. Fair, F.F. Lange. Effect of interparticle potential on forming solid, spherical agglomerates during drying. J Am Ceram Soc 87, 4-9, 2004

28. N. Tsapis, E.R. Dufresne, S.S. Sinha, C.S. Riera, J.W. Hutchinson, L. Mahadevan, D.A. Weitz. Onset of buckling in drying droplets of colloidal suspensions. Phys Rev Letters 94, 018302, 2005.

29. W-N. Wang, I.W. Lenggoro, K. Okuyama. Dispersion and aggregation of nanoparticles derived from colloidal droplets under low-pressure conditions. J Colloid Interface Sci 288, 423-432, 2005.

30. D. Sen, O. Spalla, O. Tache, P. Haltebourg, A. Thill. Slow drying of a spray of nanoparticles dispersion. In situ SAXS investigation. Langmuir 23, 42964302, 2007.

31. C.S. Kim, K.W. Ahn, S.C. Rah, S-G. Kim. Preparation of silica nanostructures spheres by sol spray drying. Drying Technol 26, 260-264, 2008.

32. A. Omrane, S. Santesson, M. Alden, S. Nilsson. Laser techniques in acoustically levitated micro droplets. Lab on a Chip - Miniaturisation for Chemistry and Biology 4, 287-291, 2004.

33. A.L. Yarin, G. Brenn, O. Kastner, D. Rensink, C. Tropea, C. Evaporation of acoustically levitated droplets. J Fluid Mechanics 399, 151-204, 1999.

34. O. Kastner, G. Brenn, D. Rensink, C. Tropea, A.L. Yarin. Investigation of the drying behaviour of suspension droplets in an acoustic tube levitator. Proceedings of the 16th Annual Conference on Liquid Atomization and Spray Systems (ILASS Europe), Darmstadt, Alemania. 2000.

35. O. Kastner, G. Brenn, D. Rensink, C. Tropea. Mass transfer from multiphase droplets during drying in a tube levitator. Proceedings of the 8th International Conference on Liquid Atomization and Spray Systems (ICLASS), Pasadena, EEUU, 2000.

36. O. Kastner, G. Brenn, C. Tropea. The drying time of single suspension droplets under various conditions. Proceedings of the Conference "Spray Drying and Related Processes", Dortmund, Alemania, 2001.

37. A.L. Yarin, D.A. Weiss, G. Brenn, D. Rensink. Acoustically levitated drops: drop oscillation and break-up driven by ultrasound modulation. Int J Multiphase Flow 28, 887-910, 2002.

38. A.L Yarin, G. Brenn, O. Kastner, C. Tropea. Drying of acoustically levitated droplets of liquid-solid suspensions: evaporation and crust formation. Phys Fluids 14, 2289-2298, 2002.

39. H. Schiffter, G. Lee. Single-droplet evaporation kinetics and particle formation in an acoustic levitator. Part 1: evaporation of water microdroplets assessed using boundary-layer and acoustic levitation theories. J Pharm Sci 96, 2274-2283, 2007

40. H. Schiffter, G. Lee. Single-droplet evaporation kinetics and particle formation in an acoustic levitator. Part 2: drying kinetics and particle formation from microdroplets of aqueous mannitol, trehalose, or catalasa. J Pharm Sci 96, 2284-2295, 2007.

41. E. Wulsten, G. Lee. Surface temperature of acoustically levitated water microdroplets measured using infra-red thermography. Chem Eng Sci 63, 5420-5424, 2008.

42. R. Kumar, E. Tijerino, A. Saha, S. Basu. Structural morphology of acoustically levitated and heated nanosilica droplet. Appl Phys Letters 97, 123106, 2010.

43. A. Saha, S. Basu, C. Suryanarayana, R. Kumar. Experimental analysis on thermo-physical processes in acoustically levitated heated droplets. Int J Heat Mass Trans 53, 5663-5674, 2010

44. A. Saha, S. Basu, R. Kumar. Particle image velocimetry and infrared thermography in a levitated droplet with nanosilica suspensions. Exp Fluids 52, 795-807, 2011.

45. R. Mondragón, L. Hernández, J.E. Juliá, J.C. Jarque, S. Chiva, B. Zaitone, C. Tropea. Study of the drying behavior of high load multiphase droplets in an acoustic levitator at high temperature conditions. Chem Eng Sci 66, 2734-2744, 2011

46. R. Mondragón, J.C. Jarque, J.E. Juliá, L. Hernández, A. Barba. Effect of slurry properties and operational conditions on the structure and properties of porcelain tile granules dried in an acoustic levitator. J Eur Ceram Soc 32, 59-70, 2012

47. R. Mondragón, J.E. Juliá, A. Barba, J.C. Jarque. Microstructure and mechanical properties of grains of silica nanofluids dried in an acoustic levitator. J Eur Ceram Soc 32, 4295-4304, 2012.

48. R. Mondragón, J.E. Juliá, L. Hernández, J.C. Jarque. Modelling of drying curves of silica nanofluid droplets dried in an acoustic levitator using the
Reaction Engineering Approach (REA) model. Drying Technol 31, 439-451, 2013.

49. R. Mondragón, J.E. Juliá, L. Hernández, J.C. Jarque. Influence of the particle size on the drying kinetics of single droplets containing mixtures of nanoparticles and microparticles. Modeling and pilot-scaled validation. Drying Technol, DOI: 10.1080/07373937.2012.757233, 2013.

50. R. Mondragón, J.E. Juliá, A. Barba, J.C. Jarque. Influence of the particle size on the microstructure and mechanical properties of grains containing mixtures of nanoparticles and microparticles. Levitator tests and pilotscaled validation. . J Eur Ceram Soc 33, 1271-1280, 2013.

51. Y. Sano, R.B. Keey. The drying of a spherical particle containing colloidal material into a hollow sphere. Chem Eng Sci 37, 881-889, 1982.

52. C. Fyhr, I.C. Kemp. Comparison of different drying kinetic models for single particles. Drying Technol 16, 1339-1369, 1998.

53. R. Vehring, W.R. Foss, D. Lechuga-Balesteros. Particle formation in spray drying. Aerosol Sci 38, 728-746, 2007.

54. C.S. Handscomb, M. Kraft, A.E. Bayly. A new model for the drying of droplets containing suspended solids after shell formation. Chem Eng Sci 64, 228-246, 2009.

55. C.S. Handscomb, M. Kraft, A.E. Bayly. A new model for the drying of droplets containing suspended solids. Chem Eng Sci 64, 628-637, 2009.

56. C.S. Handscomb, M. Kraft. Simulating the structural evolution of droplets following shell formation. Chem Eng Sci 65, 713-725, 2010.

57. M. Mezhericher, A. Levy, I. Borde. Modelling the morphological evolution of nanosuspension droplet in constant-rate drying stage. Chem Eng Sci 66, 884-896, 2011.

58. K.C. Patel, X.D. Chen. Prediction of spray-dried product quality using two simple drying kinetics models. J Food Process Eng 28, 567-594, 2005.

59. M. Mezhericher, A. Levy, I. Borde. Theoretical models of single droplet drying kinetics: a review. Drying Technol 28, 278-293, 2010.

60. T.A.G. Langrish, T.K. Kockel. The assessment of a characteristic drying curve for milk powder for use in computational fluid dynamics modelling. Chem Eng J 84, 69-74, 2001.

61. M.W. Woo, W.R. Wan Daud, A.S. Mujumdar, M.Z. Meor Talib, W.Z. Hua, S.M. Tasirin. Comparative study of droplet drying models for CFD modelling. Chem Eng Res Design 86, 1038-1048, 2008.

62. X.D. Chen, S.X.Q. Lin. Air drying of milk droplet under constant and timedependent conditions. AIChE J51, 1790-1799, 2005.

63. X.D. Chen, G.Z. Xie. Fingerprints of the drying behaviour of particulate or thin layer food materials established using a reaction engineering model. Food and Bioproducts Processing 75, 213-222, 1997.

64. X.D. Chen. The basics of a reaction engineering approach to modelling air-drying of small droplets or thin-layer materials. Drying Technol 26 , 627-639, 2008

65. X.D. Chen, S.X.Q. Lin. Air drying of milk droplet under constant and timedependent conditions. AIChE J 51, 1790-1799, 2005.

66. S.X.Q. Lin, X.D. Chen. A model for drying and aqueous lactose droplet using the reaction engineering approach. Drying Technol 24, 1329-1334, 2006

67. S.X.Q. Lin, X.D. Chen. The reaction engineering approach to modelling cream and whey protein concentrate droplet drying. Chem Eng Proces 46, 437-443, 2007.

68. M. Mezhericher, A. Levy, I. Borde. Theoretical drying model of single droplets containing insoluble or dissolved solids. Drying Technol 25, 10351042, 2007.

69. M. Mezhericher, A. Levy, I. Borde. Modelling of particles breakage during drying. Chem Eng Proces 47, 1404-1411, 2008.

70. K.C. Patel, X.D. Chen, S.X.Q. Lin. A composite reaction engineering approach to drying of aqueous droplets containing sucrose, maltodextrin (DE6) and their mixtures. AIChE J 55, 217-231, 2009.

71. N. Fu, M.W. Woo, S.X.Q. Lin, Z. Zhou, X.D. Chen. Reaction engineering approach (REA) to model the drying kinetics of droplets with differen initial sizes-experiments and analyses. Chem Eng Sci 66, 1738-1747, 2011.

Recibido: $14 / 05 / 2013$

Recibida versión corregida: 25/07/2013

Aceptado: 25/07/2013 